\title{
Increasing the efficiency of highly concentrated coal-water fuel based on the simulation of non-Newtonian fluid flow
}

\author{
Nataliya Chernetskaya-Beletskaya ${ }^{1}$, Andriy Rogovyi ${ }^{2}$, Igor Baranov ${ }^{1,{ }^{*}}$, Alexander Krut $^{3}$, Maria Miroshnikova ${ }^{1}$, and \\ Nikita Bragin $^{1}$ \\ ${ }^{1}$ Volodymyr Dahl East Ukrainian National University, Department of logistics management and traffic safety vehicle, 93400 \\ Severodonetsk, pr. Central 59-a, Ukraine \\ ${ }^{2}$ Kharkiv National Automobile and Highway University, Department of theoretical mechanics and hydraulics, 61002 Kharkiv, \\ Yaroslava Mudrogo Str, 25, Ukraine \\ ${ }^{3}$ State scientific research, design and design institute of coal industry "Ukrndiproekt", 03142 Kyiv, Academician Palladin Avenue, \\ 46/2, Ukraine
}

\begin{abstract}
The analysis of further prospects for increasing the efficiency of transportation of coal-water fuel in hydro-transport systems of industrial enterprises is carried out. The mathematical model of the spatial three-dimensional flow of coal-water fuel was developed on the basis of SST turbulence model based on the solution of Navier-Stokes equation. As a result of the calculation, the values of pressure loss, flow rate and velocity distribution over the cross section of the pipeline in the straight section and in the turn were determined, which allowed determining the energy consumption during coal-water fuel transportation in the industrial hydro-transport system. The performed studies allowed us to refine the mathematical model of water-coal suspension flow and, thus, improve the patterns of influence of hydrotransportation scheme and parameters of coal-water fuel on energy consumption for its supply to enterprise consumers. By means of mathematical model of non-Newtonian fluids flow, the patterns of influence of hydro-transport system parameters and transportation modes of coal-water fuel on its energy indicators in industrial hydro-transport systems are determined. The obtained results are related to reduction of energy consumption, optimization of enterprise transport network configuration and increase of efficiency of coal-water fuel transportation to enterprise energy facilities.
\end{abstract}

\section{Introduction}

The sustainable development of fuel and energy complex of Ukraine is associated with the use of alternative fuels, which are the most efficient and cost-effective for the energy sector of the country. The enterprises of thermal power and coal mining complex of Ukraine: mines and concentrating factories - have in their structure boilerhouses working on coal with the total annual demand of the enterprise up to 150 thousand tones. Meanwhile, further prospects for the use of coal fuels in Ukraine are inextricably connected with the use of coal-water fuel (CWF).

Coal-water fuels are widely used in such countries as China, USA, Russia, Italy, Sweden, Japan and Canada. Industrial hydro-transport systems (HTS) used for transportation of CWF have a wide application range: power industry, metallurgy, construction materials production, chemical industry. CWF consumers can be small, medium and large industrial enterprises of thermal energy and coal-mining complex: mines and concentrating factories. Industrial HTS (IHTS) can be transport arteries of fuel and energy or fuel and metallurgical complexes; can supply CWF to heating, heating and production and production boiler houses on the enterprise territory.

Increasing the efficiency of CWF transportation in IHTS means reducing the cost of transportation of $1 \mathrm{tkm}$ of cargo at a constant cargo flow, at this the cost of transportation directly depends on transportation energy consumption. The disadvantage of pipeline hydrotransport is the increase in energy consumption, depending on the proportions of various fractions of granulometric composition (GMC) of the transportation product, as a result, in case of inappropriate composition, energy consumption for CWF transportation increases by up to $1.5-1.7$ times [1].

\subsection{Formulation of the problem}

The problem of the functioning of IHTS is the absence of a specific criterion for assessing GMC of highly concentrated CWF (HCCWF) and that there are no recommendations how to prepare and adhere to appropriate proportions of its various fractions, resulting in increased energy consumption when transporting CWF.

\footnotetext{
*Corresponding author: baranov_90@ukr.net
} 
The absence of CWF transportation scheme optimized according to technical and economic indicators and justified in terms of minimization of energy consumption of the parameters of the enterprise operation leads to an increase in the cost of CWF transportation by $19 \%$. In addition, in order to estimate the energy consumption of HCCWF transportation and to increase the efficiency of HTS operation, it is necessary to clarify the flow patterns along the pipeline and to improve the mathematical models of process description of HCCWF transportation, which is a relevant scientific and practical task.

A significant number of theoretical and experimental studies of CWF transportation confirms the strong impact of HTS on energy efficiency of transportation process $[2,3]$.

The study of impact of various HTS options on CWF transportation parameters requires a large number of experimental studies providing for significant investment.

Presently, it is possible to perform the set task using the numerical methods of fluid flow problems, which are characterized by a minimum time consumption for calculation $[4,5]$.

This approach allows us to reduce the basic number of experimental studies by replacing some of the expensive experimental research with theoretical numerical ones.

To implement this approach it is necessary to validate mathematical models in order to obtain a minimum difference between the results of mathematical modelling and experimental research $[6,7]$.

Calculations on the mathematical models of CWF transportation processes in the pipeline and various elements of HTS configuration can reduce the time for improving the calculation methods of hydrotransportation parameters, obtain the most accurate characteristics of the systems, and define the limits of their use [8,9]. Therefore, mathematical modelling is one of the most important and relevant tasks of HCCWF flow research [10].

\subsection{Analysis of recent research and publications}

In recent years, a significant number of works have been devoted to the simulation of Bingham fluids flow [2, 3, $5,8,9,11,12,13,14]$. The works $[11,12]$ are devoted to the flow simulation of coal-water mixtures (CWM) in particular, but for the coal of Chinese and Indian regions. At the present time, transportation studies of HCCWF for the coal of fuel and energy complex of Ukraine, have not been conducted.

In $[11,14]$ the simulation of non-Newtonian fluids flow in the turns was performed, but regressive dependencies for all variants of rheological properties were not given. In article [15] the exact calculation of characteristics of the fluid flow are given, but only by a pipeline, which does not allow using the obtained correlation for various options of HTS configuration.

As the analysis of the research has shown, the greatest influence on transportation parameters of CWF in IHTS have the characteristics of raw coal, concentration and HTS, as well as the transportation velocity parameters, on which the rheological properties and energy consumption depend on the whole $[2,3,5$, 13].

\section{Mathematical model of non-Newtonian fluids flow}

In this paper, we used the SST turbulence model, which proved to be the best in the study of virtually any wall-bounded flow. When using the SST model, there appear significant errors in determining the velocity and pressure values at certain points, but the integral parameters are determined with its help with adequate accuracy and the error does not exceed 10\% [16-19].

Calculation errors naturally arise in flow separation zones. However, given the computational appeal of this model in comparison with direct numerical simulation (DNS), the methods of large eddy simulation (LES) and detached eddy simulation (DES), the SST model has become virtually universal for uncomplicated flows. To solve this problem, Reyonolds averaged Navier-Stokes equations and continuity equations were used:

$$
\begin{aligned}
& \frac{\partial u_{i}}{\partial t}+u_{j} \frac{\partial u_{i}}{\partial x_{j}}=F_{i}-\frac{1}{\rho} \frac{\partial p}{\partial x_{i}}+\frac{\partial}{\partial x_{j}}\left[\frac{\mu_{e f}}{\rho} \frac{\partial u_{i}}{\partial x_{j}}\right] \\
& \frac{\partial u_{i}}{\partial x_{i}}=0
\end{aligned}
$$

where $x_{j}-$ Cartesian coordinates; $u_{j}-$ velocity projections in Cartesian coordinate system; $\mu_{e f}=\mu+\mu_{t}$ effective viscosity; $\mu_{t}-$ turbulent viscosity; $\mu-$ molecular viscosity.

System of equations of SST model [20]:

$$
\begin{aligned}
& \frac{\partial(\rho k)}{\partial t}+\frac{\partial}{\partial x_{j}}\left(\rho u_{j} k\right)=\frac{\partial}{\partial x_{j}}\left(\mu_{e f} \frac{\partial k}{\partial x_{j}}\right)+P_{k}-\beta^{*} \rho k \omega ; \\
& \frac{\partial(\rho \omega)}{\partial t}+\frac{\partial}{\partial x_{j}}\left(\rho u_{j} \omega\right)=\frac{\partial}{\partial x_{j}}\left(\mu_{e f} \frac{\partial \omega}{\partial x_{j}}\right)- \\
& -\rho \beta \omega^{2}+C d_{\omega}+\alpha \frac{\rho}{\mu_{t}} P_{k},
\end{aligned}
$$

where $k$ - kinetic energy of turbulent pulsations; $\omega-$ specific dissipation; $P_{k}-$ generated member; $C d_{\omega}-$ a cross member. Constants and descriptions of equations can be found in [20].

The modelling of the rheological parameters of Bingham fluid was carried out according to the formula $[12,21]$ :

$$
\mu=\frac{\tau_{0}}{\gamma}+\eta
$$

where $\tau_{0}$ - initial yield stress, $\gamma$ - shear rate between fluid layers, $\eta$ - structural viscosity.

Control volume approach and PISO algorithm (Pressure Implicit with Splitting of Operators) were 
used. Numerical schemes of the second order were used. The calculations were carried out until the residual in all the main parameters did not reach the value of $10^{-5}$. In addition, the second condition for stopping the calculations was time invariance of costs. The constancy criterion of cost is the value of $1 \%$.

In order to form the flow in the boundary layer prismatic elements were used. To determine a minimal sufficient flow grid, calculations on three different grids were made and the grid with such a number of elements from which the integral parameters cease to change (the number of elements of 470 thousand) was selected. During the preliminary calculations, the effect of the parameter $y^{+}$on the solution accuracy was also estimated. Thus, based on the calculations as well as recommendations given in [22], it was decided to use the grids with $y^{+}<4$.

\subsection{Calculation results of coal-water fuel}

At the moment, there is quite a number of software products that allow you to calculate the flow parameters of fluids and gases: OpenFoam, Ansys Fluent, Cosmos FlowWorks, Ansys CFX, FlowVision and many others. In this paper, the calculation was made using the ANSYS CFX (Academic Student Licence with noncommercial use only) software based on the Bingham model.

To verify the numerical calculations, experimental studies of CWF flow by direct pipeline were performed and pressure losses for realizing the given loss or CWF velocity were found [23]. Test bench for physical examination is equipped with measuring equipment with sufficient accuracy for calculations verification and then for adequacy verification. The maximum error of determining the pressure losses did not exceed $1 \%$.

As a result of the calculations, the values of pressure losses, flow rate and velocity distribution at the intersection of the pipeline in the straight part and in $90^{\circ}$ turn (Fig. 1) were determined, which allowed us to determine energy costs when transporting CWF in IHTS. In stationary calculation, the trajectory of liquid particles flow has the form of flow lines, which coincide with the trajectories of particles.

As Fig. 1 shows, near the pipe axis there is a zone of approximately the same velocity, which qualitatively coincides with the results of experimental studies. This zone is a core flow, which is typical of Bingham fluids flow.

Thus, it may be affirmed that the numerical calculation results qualitatively coincide with the analytical description of Bingham flow and the results of experimental research. Because of the fact, that flow lines are straight lines, as well as when checking Reynolds numbers, it can be concluded that the flow is laminar. Therefore, calculations and comparison with experimental data of different models were performed: laminar, $k-\varepsilon$ and SST model (Fig. 2). The figure shows the error in calculating the pressure loss in percentages. As can be seen from the figure, the largest error is the flow calculation without taking into account the rheological law, which is the flow of the Newtonian fluid with the same viscosity. The laminar model and SST model, taking into account the rheological law, have the least error.

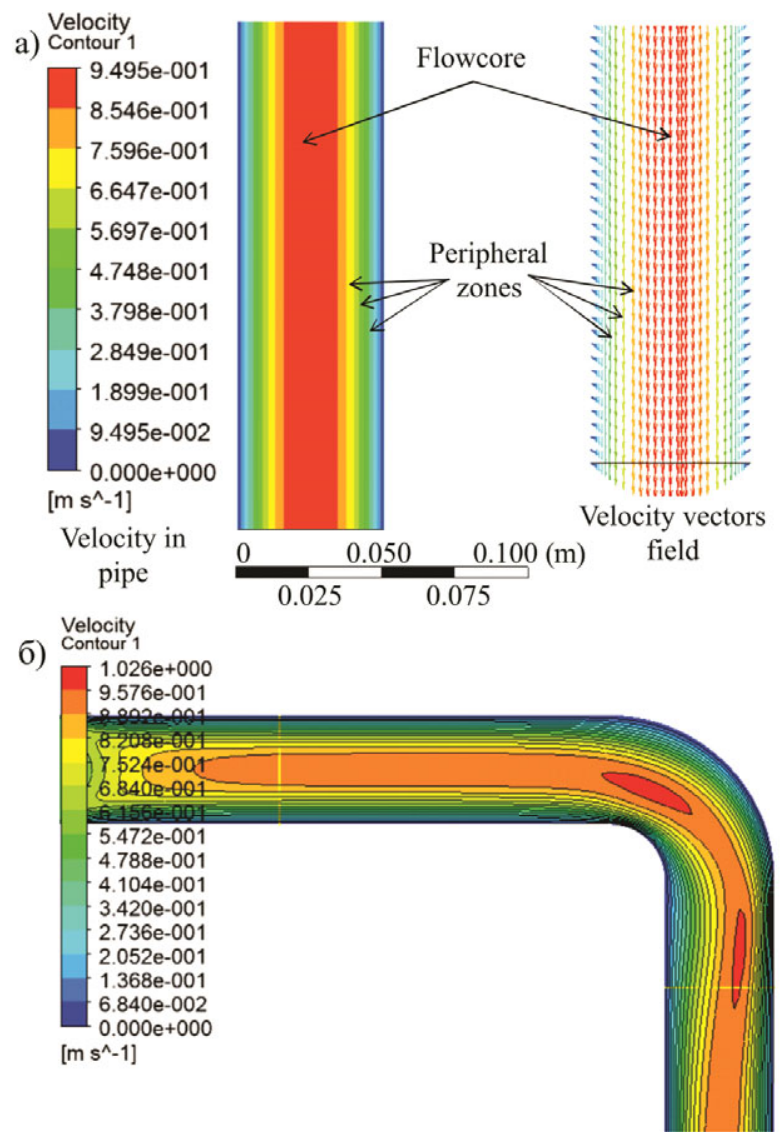

Fig. 1. Graphic representation of calculation results of CWF flow based on Menter SST model: a) - straight section (laminar flow); б) - shaped part of the pipeline (flow with elements of turbulence).

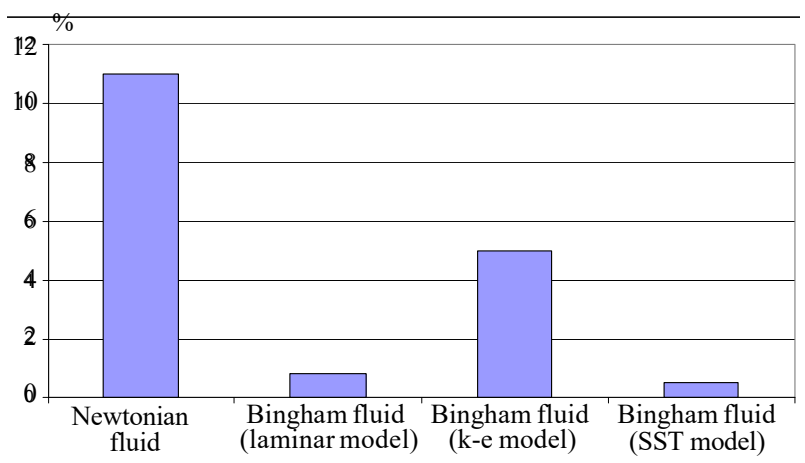

Fig. 2. Comparison of the accuracy of determining pressure loss according to different models.

According to the comparison of the calculation accuracy, it is of no particular importance, which model to select for calculation, laminar or Menter model, but regarding the versatility of model use, the SST model is more versatile and can be used for any flow type.

To verify the selected model, calculations of Bingham fluid flow of CWF with different parameters of rheological model are performed, the results of which are shown in Fig. 3. Here the calculation error of pressure losses in pipe at a different average velocity is 
presented. The error is calculated by comparing the results of theoretical studies with the experimental data.

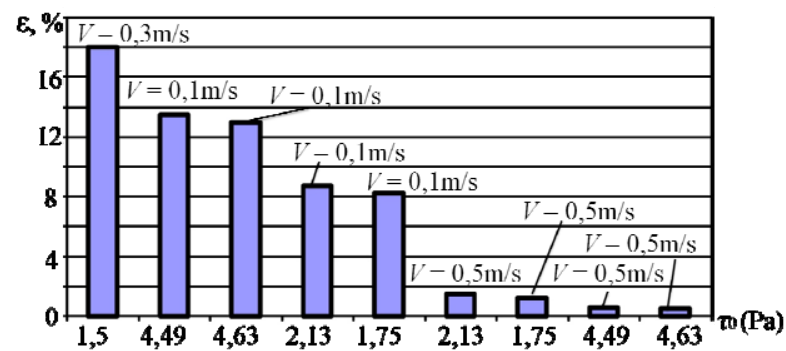

Fig. 3. Calculation error of pressure loss at Bingham fluid flow using SST-model.

As Fig. 4 shows, flow calculations with Reynolds numbers $\operatorname{Re}>30$, have the least error, that is, the greater the velocity of Bingham fluid is, the less is the calculation error according to SST-model of turbulence. In general, the error does not exceed $20 \%$, and with an increase in velocity makes $0,5 \%$, which is an acceptable result, and suggests that in the future, you can use the software package and SST model for calculation of CWF flow and use a mathematical model for optimization of fuel granulometric composition.

Granulometric composition (GMC) of CWF influences the viscosity of the rheological model, which further has an effect on the velocity profile and pressure losses of fuel flow (Fig. 4).

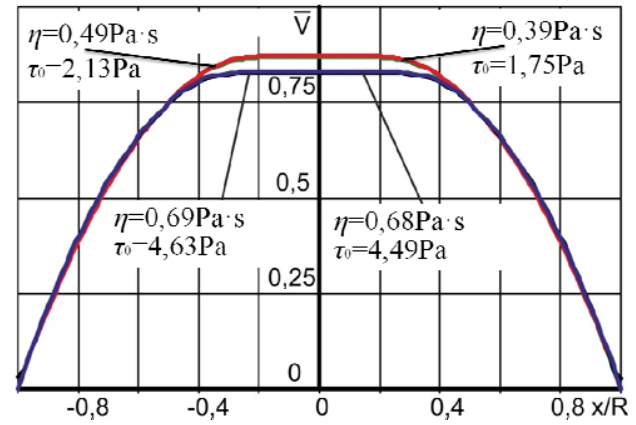

Fig. 4. Velocity profiles of CWF flow of different GMC.Fig. 4. Shows the velocity profiles obtained when calculating CWF of different GMC. With an increase in the initial shear stress, the maximum flow velocity decreases. In Fig. 4 velocity is assigned to the maximum velocity calculated according to Newtonian fluid model. To find the optimal values of initial shear stresses and structural viscosity, a numerical experiment on the basis of experiment planning methods was conducted in order to reduce pressure losses [21].

\subsection{Calculation of coal-water fuel flow in bends}

In the shaped elements of different configuration (elbows, bends) due to the change of flow structure, there appear centrifugal forces, directed from the center of the curvature to the outer wall of the pipeline. That is the reason for a pressure increase at the outer wall and its decrease at the inner wall during the transition of the flow from the straight pipeline section to the curved one.
When designing HTS and optimizing CWF flow, it is necessary to determine the pressure losses at bends with curvature radius of the standard values.

Fig. 5-7 show the velocity distribution of coal-water fuel in a bend with curvature radius $\bar{R} \cdot=1,0$ at different Reynolds numbers with rheological properties: $\eta=0,68$ $\mathrm{Pa} \cdot \mathrm{s}, \tau_{0}=4,49 \mathrm{~Pa}$.

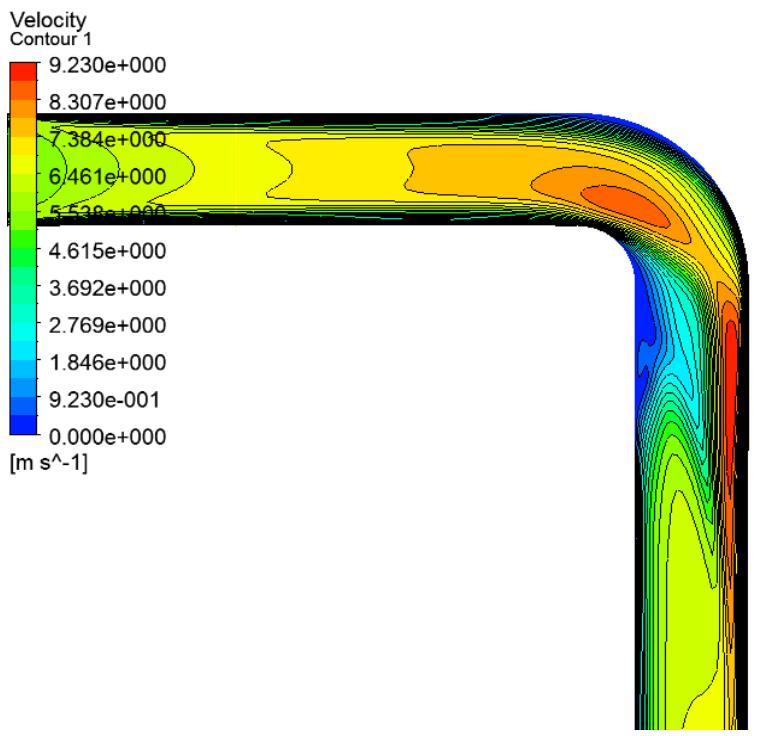

Fig. 5. Velocity distribution of CWF flow at $90^{\circ}$ turn with curvature radius $\bar{R}=1,0, \operatorname{Re}=440$.

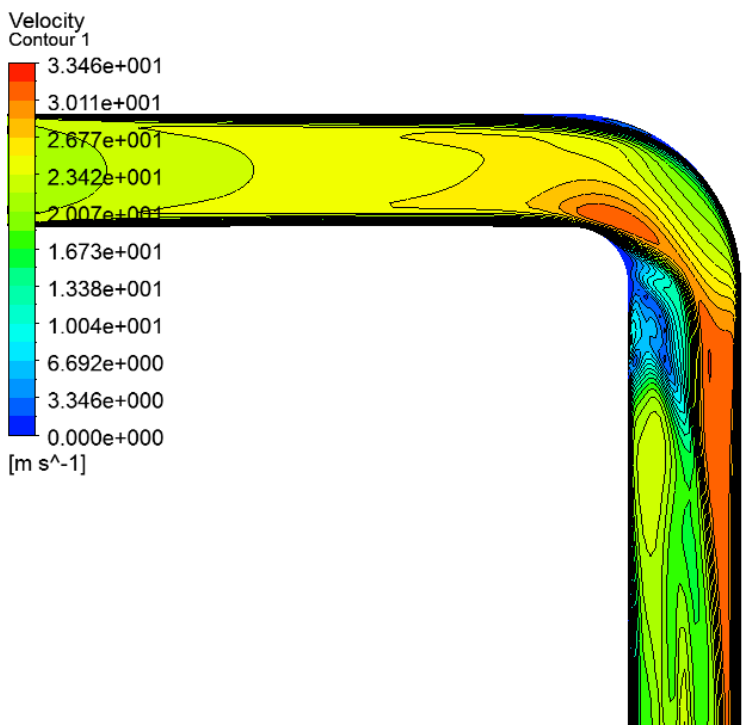

Fig. 6. Velocity distribution of CWF flow at $90^{\circ}$ turn with curvature radius $\bar{R}=1,0, \operatorname{Re}=1840$

With the increase of Reynolds numbers, there is a flow separation after the turn and an increase in hydraulic resistance coefficient [24]. Fig.8 shows the dependence of pressure losses in the bend with curvature radius $\bar{R}=1,0$ depending on the average velocity of CWF with rheological properties: $\eta=0,68 \mathrm{~Pa} \cdot \mathrm{s}, \tau_{0}=4,49 \mathrm{~Pa}$.

Fig. 9 shows a monotonous decrease in hydraulic resistance coefficient $\varsigma=2 \Delta p / \rho V^{2}$ of a bend with $\bar{R}=$ 1,0 with Reynolds number increase. 


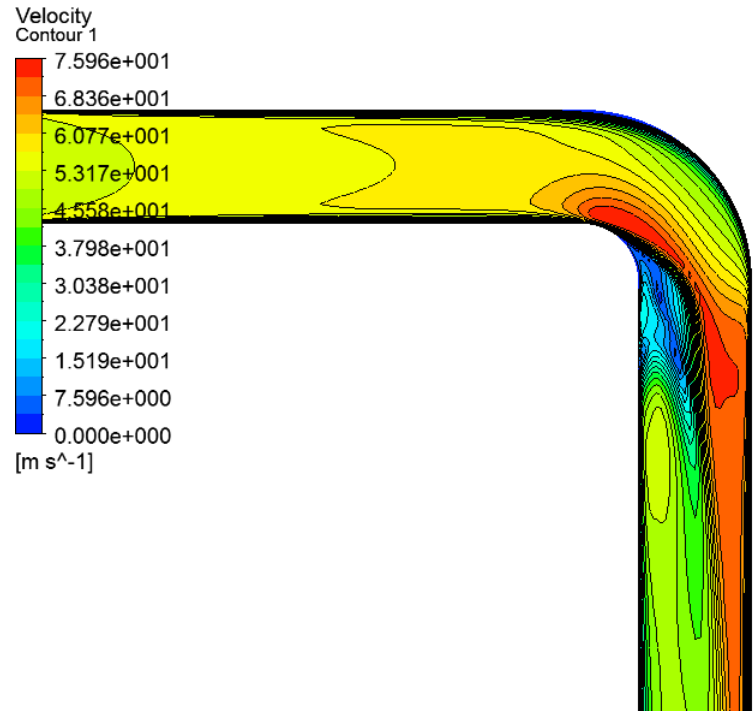

Fig. 7. Velocity distribution of CWF flow at $90^{\circ}$ turn with curvature radius $\bar{R}=1,0, \mathrm{Re}=4600$.

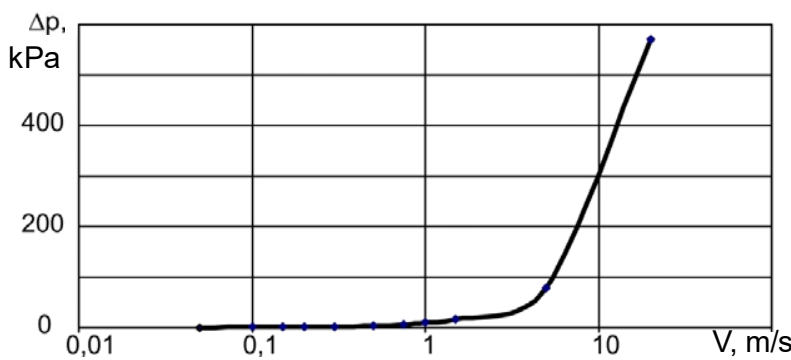

Fig. 8. Dependence of pressure losses in a bend with $\bar{R} \cdot=1,0$ on average flow velocity.

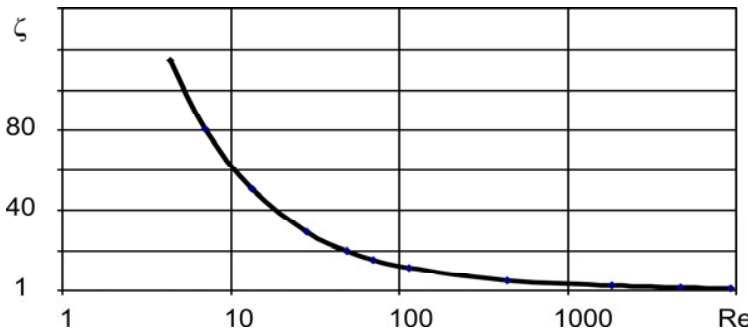

Fig. 9. Dependence of hydraulic resistance coefficient in a bend with $\bar{R}=1,0$ on Reynolds number.

Coefficient $\varsigma$ tends to a value of 1.0 with Reynolds number increase to value $\operatorname{Re}>1000$ [25]. For Newtonian fluid coefficient $\varsigma$ of such a bend is equal to 0.21 for smooth walls, which is almost five times less than for CWF with the stated rheological properties.

In order to take into account the hydraulic resistance of hydro-transport system sections, which include shaped parts, the following expression for specific pressure losses is used at the next stages of work

$$
i=i_{s}+\frac{1}{2 \ell_{\partial}} \sum_{j=1}^{n}\left[\kappa_{j} \zeta_{j} \rho V^{2}\right]
$$

where $i_{l}-$ specific resistance of the straight part of section, $\mathrm{Pa} / \mathrm{m} ; l_{\partial}-$ length of the section; $j$ - index of the type of shaped part of the pipeline; $n$ - number of types of shaped parts, which are part of the section; $\kappa_{j}-$ number of shaped parts of $\mathrm{j}$-type; $\zeta_{j}$ - coefficient of local resistance of shaped part of $j$-type.

\section{Influence of granulometric composition of coal-water fuel on pressure losses}

To obtain dependencies that have an effect on pressure loss during CWF transportation, a serious of calculations has been carried out with the helps of the proposed mathematical model for different GMC. It is established that the most important factors influencing the parameters of CWF hydro transportation are the concentration of the solid component (C) and the parameters characterizing the bimodality of GMC of CWF solid phase. This approach allows comparing the pressure loss for various options of bimodal composition and makes it possible to trace $\Gamma_{1}, \Gamma_{2}$ effect at different concentrations on CWF hydraulic parameters.

During the simulation, the following parameters are selected: $D=50 \mathrm{~mm}, V=0.5 \mathrm{~m} / \mathrm{s}$.

For different $\Gamma_{1}, \Gamma_{2}$ on the basis of experimental research, rheological properties $\eta$ and $\tau_{0}$ were determined, and introduced into the calculation of the mathematical model.

Fig. 10 shows that pressure losses curves, depending on $\Gamma_{1} / \Gamma_{2}$ ratio (bimodality criterion $(\mathrm{BC})$ have clearly defined minimum in the range of 1.7...2.0. In order to confirm the obtained conclusions regarding the minimum of hydraulic pressure losses during CWF transportation by pipeline, a comparison with experimental data was made, which is presented in Fig. 11.

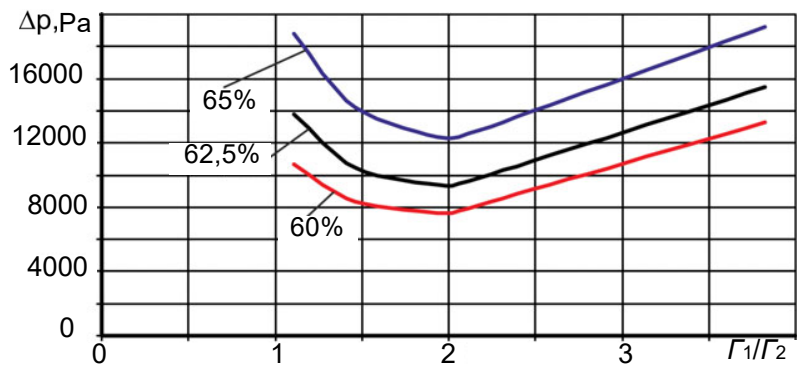

Fig. 10. Dependence of pressure losses on CWF granulometric composition.

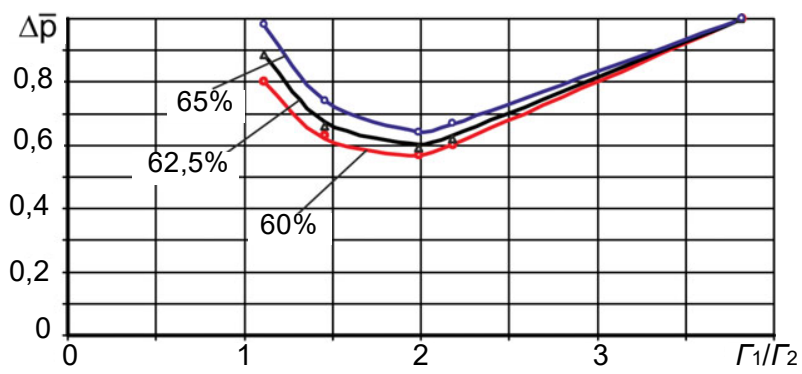

Fig. 11. Comparison of numerical calculation results with experimental results.

Pressure losses shown in Fig. 11 are attributed to the maximum pressure losses corresponding to losses during 
transportation of fluid with GMC having the ratio $\Gamma_{1} / \Gamma_{2}=3.82$. The lines correspond to the numerical calculations, the points correspond to the experimental studies. The difference between the experimental data and the numerical calculation does not exceed $1.6 \%$.

Thus, as it can be seen from Fig.11, with an increase in concentration, the relative pressure losses increase. Minimal losses correspond to the GMC with an index $\Gamma_{1} / \Gamma_{2}=1.7 \ldots 2.0$. In addition, in [1] the division of bimodal variants of CWF into variants is proposed: those having a harmonious structure and those, which are inherently imperfect.

According to the estimated number of layers with a diameter, equivalent to particles of fine fraction, the number of such layers in the range of 2.2-2.6 should correspond to the harmonious structure. According to the results of the study [26], the use of GMC of CWF (with $\Gamma_{1} / \Gamma_{2}=2.33$ ), allows reducing its transportation energy consumption to the HTS of the company "DTEK Dobropilvugillya LLC" by 86.13 thousand UAH/year compared with random granulometric composition, which was chosen as an output one.

As a result of using sequential-beam transportation of CWF, the total cost of pipeline laying has decreased by 893.89 thousand UAH in comparison with beam scheme. The effect of optimizing the topology of the transport network of distribution by CWF consumption points, optimizing the total pipeline costs, equipment and electric energy for transportation and selection of GMC of CWF amounted to 183.75 thousand UAH/year [26].

The total volume of capital investments in the proposed HTS project amounted to 7.47 million UAH, annual operating expenses amount to 199.89 thousand UAH. The overall economic effect of introducing IHTS instead of road transport is 4.08 million UAH/year, payback period of the project is 1.83 years. When using pipeline transport for CWF transportation, as HTS of the enterprise, the difference between capital costs is 0.34 million UAH, and operating costs are by 4.1 million $\mathrm{UAH} /$ year lower compared to road transport.

\section{Conclusion}

1. It is determined that further prospects for increasing the efficiency of CWF transportation in HTS of industrial enterprises are based on optimization of granulometric composition of solid fuel component and its concentration.

2. The performed theoretical studies allowed refining the mathematical model of CWS flow in IHTS and thus improve the patterns of influence of hydro-transport scheme and CWF parameters on energy consumption of coal fuel supply to consumers.

3. The developed mathematical model of the nonNewtonian fluids flow allowed us to determine the flow structure and energy loss during CWF transportation in IHTS and optimize the configuration of transport network, taking into account patterns of influence of $\mathrm{GMC}$, rheological properties and transportation modes on transportation energy consumption.
4. It was established that according to the results of modelling, the specific pressure losses of transported by pipeline CWF have a local minimum in the studied range of change of the proposed criterion.

5. According to the results of theoretical studies of CWF flow taking into account the bimodality criterion and its rheological properties, the following conclusions can be made:

- According to flow numerical calculation results, a zone of approximately the same velocity is obtained near the pipe axis, which qualitatively coincides with the results of experimental studies. The results of numerical calculation qualitatively coincide with the analytical description of Bingham flow and the results of experimental studies.

- The curves of the pressure losses, depending on $\Gamma_{1} / \Gamma_{2}$ ratio, have a clearly expressed minimum in the range of 1.7...2.0. The difference between the experimental data and the numerical calculation does not exceed $1.6 \%$ for formed dependencies of the loss on $\Gamma_{1} / \Gamma_{2}$. With an increase in concentration, relative loss of pressure increases.

- Hydraulic resistance coefficient $\varsigma$ of a bend with $\bar{R}^{\cdot}=1,0$ monotonously decreases with increasing Reynolds number and tends to values of 1.0 with Reynolds number increase to values $\mathrm{Re}>1000$. For Newtonian fluid coefficient $\varsigma$ of such a bend is equal to 0.21 for smooth walls, which is almost five times less than for CWF with stated rheological properties.

\section{References}

1. I.O. Baranov. Improving the highly loaded coalwater slurry fuel transportation efficiency in the hydro-transport systems of industrial enterprises. Abstract thesis for degree candidate of technical sciences. (Doctor of Philosophy), speciality 05.22.12 - Industrial Transport (275 - Transport Technology). - V. Lazarian Dnipropetrovsk National University of Railway Transport, Dnipro, 21 (2019).

2. T. Norton, D. Sun. Computational fluid dynamics (CFD)-an effective and efficient design and analysis tool for the food industry: a review. Trends in Food Science \& Technology, 17(11), 600-620 (2006).

3. M. Sorgun, M. Ozbayoglu. Predicting frictional pressure loss during horizontal drilling for nonNewtonian fluids. Energy Sources, Part A: Recovery, Utilization, and Environmental Effects, 33(7), 631-640 (2011).

4. D. Syomin, A. Rogovyi. Features of a working process and characteristics of irrotational centrifugal pumps. Procedia Engineering, 39, 231237 (2012).

5. R. Peyret, T. Taylor. Computational methods for fluid flow. (New York: Springer Science \& Business Media) (2012).

6. E. Fjodorov. Metody izmerenija urovnja $i$ granic razdela mnogofaznyh zhidkih sred. Materialy VI 
nauchno-prakticheskoj konferencii 27-30 maja 2015 g. - Tomsk, 65 (2015). [in Russian]

7. A. Thompson. Basic hydrodynamics. Elsevier Science. Library of Congress Cataloging in Publication Data, 177 (2013).

8. J. Wang, S. Wang, T. Zhang, F. Battaglia. Mathematical and experimental investigation on pressure drop of heterogeneous ice slurry flow in horizontal pipes. International Journal of Heat and Mass Transfer, 108, 2381-2392 (2017).

9. F. Kabinejadian, D. Ghista. Compliant model of a coupled sequential coronary arterial bypass graft: effects of vessel wall elasticity and non-Newtonian rheology on blood flow regime and hemodynamic parameters distribution. Medical engineering and physics, 34(7), 860-872 (2012).

10. C. Hervé. The basics of plant hydraulics. The Journal of plant hydraulics, 1, 178-188 (2014).

11. M. Liu, Y. Duan. Resistance properties of coalwater slurry flowing through local piping fittings. Experimental Thermal and Fluid Science, 33(5), 828-837 (2009).

12. A. Rawat, S. Singh, V. Seshadri. Computational methodology for determination of head loss in both laminar and turbulent regimes for the flow of high concentration coal ash slurries through pipeline Particulate Science and Technology. - T. 34. - №. 3., 289-300 (2016).

13. K. Ekambara, R. Sanders, K. Nandakumar, J. Masliyah. Hydrodynamic simulation of horizontal slurry pipeline flow using ANSYS-CFX. Industrial \& Engineering Chemistry Research, 48(17), 81598171 (2009).

14. P. Csizmadia, C. Hös. CFD-based estimation and experiments on the loss coefficient for Bingham and power-law fluids through diffusers and elbows. Computers \& Fluids, 99, 116-123 (2014).

15. P. K. Swamee, N. Aggarwal. Explicit equations for laminar flow of Bingham plastic fluids. Journal of Petroleum Science and Engineering, 76 (3-4), 178184 (2011).

16. J. Yin, L. Jiao, L. Wang. Large eddy simulation of unsteady flow in vortex diode, Nuclear Engineering and Design T. 240. - №. 5. - 970-974 (2010).

17. A. Rogovyi. Energy performances of the vortex chamber supercharger. Energy, 163, 52-60 (2018).
18. Y. H. Alahmadi, A. F. Nowakowski. Modified shear stress transport model with curvature correction for the prediction of swirling flow in a cyclone separator Chemical Engineering Science. Vol. 147. - 150-165 (2016).

19. H. R. Thakare and A. D. Parekh. Computational analysis of energy separation in counter-flow vortex tube. Energy. Vol. 85. - 62-77 (2015).

20. P. E. Smirnov, F. R. Menter. Sensitization of the SST turbulence model to rotation and curvature by applying the Spalart-Shur correction term. Journal of Turbomachinery. Vol. 131. - №. 4. - 041010 (2009).

21. N. Chernetskaya-Beletskaya, A. Rogovyi, A. Shvornikova, I. Baranov, M. Miroshnikova, N. Bragin. Study on the coal-water fuel pipeline transportation taking into account the granulometric composition parameters. International Journal of Engineering \& Technology. - Vol. 7, Iss.4.3, 240-245 (2018).

22. G. Besagni, F. Inzoli. Computational fluid-dynamics modeling of supersonic ejectors: Screening of turbulence modeling approaches. Applied Thermal Engineering; 117: 122-144 (2016).

23. N. Chernetskaya-Beletskaya, I. Baranov, M M. Miroshnikova. Zabezpechennja stalogo rozvitku regionu: ekonomichni, upravlinski, pravovi ta informacijno-tehnichni aspekti: kolektivna monografija ; za zag. red. Ju. I. Kljus., N. V. Shvec. - Severodoneck: vid-vo SNU im. V. Dalja ISBN 978-617-11-0118-0, 193-212 (2017). [in Ukrainian]

24. N. Chernetskaya-Beletskaya, A. Rogovij, I. Baranov, M. Miroshnikova. Matematichna model prostorovoï trivimirnoï techï vodovugilnogo paliva. Visnik SNU im. V. Dalja, № 1 (242) - 159-164 (2018). [in Ukrainian]

25. N. Cherneckaya-Beleckaya, A. Rogovyi, I. Baranov, M. Miroshnykova. Mathematical model flow coalwater fuel. Globalization of scientific and educational space. Innovations of transport. Problems, experience, prospects: thesis, May 2018, Italy - Severodonetsk: Volodymyr Dahl East Ukrainian National University, 82-83 (2018).

26. I. Baranov. Formuvannja optimizacijnoï modeli viboru parametriv transportuvannja vodovugilnogo paliva promislovim gidrotransportom. Visnik SNU im. V. Dalja, № 3 (244), 7-13 (2018). [in Ukrainian] 